\title{
Second-Order Finite Elements for Hex-Dominant Explicit Methods in Nonlinear Solid Dynamics
}

\author{
Kent T. Danielson ${ }^{\mathrm{a}, 1}$, Mark D. Adley ${ }^{\mathrm{b}}$, and T. Neil Williams ${ }^{\mathrm{c}}$ \\ ${ }^{a}$ Research Group, Engineering Systems and Materials Division, CEERD-GMR \\ Kent.T.Danielson@usace.army.mil \\ ${ }^{\mathrm{b}}$ Impact and Explosion Effects Branch, CEERD-GMI \\ Mark.D.Adley@usace.army.mil \\ ${ }^{\mathrm{c}}$ Impact and Explosion Effects Branch, CEERD-GMI \\ Neil.T.Williams@usace.army.mil \\ Geotechnical and Structures Laboratory \\ U.S. Army Engineer Research and Development Center \\ 3909 Halls Ferry Road, Vicksburg, MS 39180-6199 USA
}

\begin{abstract}
Hexahedral-dominant modeling approaches strike a balance of meshing ease and accuracy/efficiency by exploiting wedge (and/or pyramid) elements to transition from hexahedral elements to volumes filled by other types. Unfortunately, first-order wedges are frequently very poor performers and are the only ones typically contained in explicit solid dynamic programs. The historic preference of first-order elements with explicit methods has frequently been for simplicity and cost, but has also been from the lack of both a satisfactory consistent nodal loading distribution and an acceptable mass lumping technique for serendipity elements. Row summation lumping, for example, produces negative and zero vertex node masses for the popular fifteen and eighteen node wedges, respectively, and zero vertex nodal loads from a uniform traction on the triangular faces. The paper first presents twenty-one node wedge element formulations that produce all positive nodal loads from uniform tractions and row summation mass lumping for this element is shown to produce all positive nodal masses. In addition, they are compatible with other second-order elements applicable to lumped mass explicit methods. Performance is assessed in standard benchmark problems and practical applications using various elastic and elastic-plastic material models and involving very large strains/deformations, severe distortions, and contact-impact. These wedge elements are evaluated on their own as well as part of hexahedral-dominant meshes that use wedges for fill regions and transition from hexahedral to tetrahedral elements. In all cases, these elements performed satisfactorily and thus demonstrate their viability and benefits for practical applications, especially for fill and transition regions of low interest.
\end{abstract}

Keywords hex-dominant meshing, finite element, wedge, higher order, explicit time integration

\section{Introduction and background}

Pentahedral prismatic "wedge" finite elements are not typically used alone in solid mechanics models, but are popular for modeling fill and transition regions in hexahedral-dominant meshes. Commonly referenced by various other names, e.g., prism, pentahedron, cake, chisel, digonal cupola,

\footnotetext{
${ }^{1}$ Corresponding Author, Telephone: 1-601-634-2039
} 
etc., the isoparametric parent element is a prism with two parallel triangular faces and three quadrilateral faces (see Fig. 1). The authors prefer the wedge designation because it is descriptive, hexahedra are also technically prisms, and pyramid elements are also pentahedra. Wedges generally do not perform as well as brick elements, but can more easily mesh certain complex areas, particularly at cylindrical axes, and their compatibility with brick elements permits direct connection to an otherwise largely hexahedral mesh. Their mixture of both quadrilateral and triangular faces permits transition between hexahedral and tetrahedral regions without the use of multipoint constraints. Pyramid elements also can make such connections, but they fill regions in a different manner and complexities in their shape functions can affect performances differently than with wedge elements, thus showing both pros and cons for both pyramids and wedges. Analysts historically have created such models directly or may use more recent automatic "Hex-Dominant" meshing approaches, e.g., [1-3], that generate only brick elements until they encounter difficult regions that they then fill with combinations of wedge, tetrahedral, and pyramid elements. It is common to make a two-dimensional mesh of quadrilaterals and a limited number of triangles, e.g., by a paving algorithm, and then sweep it in an out of plane direction so as the quadrilaterals and triangles become hexahedra and wedges, respectively. Unfortunately, first-order wedges typically are overly stiff, particularly in bending or nearly incompressible material applications, so they generally should be well-shaped and are preferred only for noncritical regions of low stress/strain gradients. Second-order wedge elements, however, perform much better in unstructured meshes for both nearly incompressible materials and structural flexure. They still are not as desirable as secondorder bricks, but second-order wedges are not as prone to the severe locking and over-stiffness as first-order ones. Unstructured tetrahedral meshing approaches can also more easily grid complicated geometries, but they do not perform any better (or as well) than wedge elements and are considerably more computationally expensive, since they generally consist of many more elements than in a hexahedral/wedge mesh (typically by one or more orders of magnitude). Hex-Dominant approaches are available in various popular meshing software, e.g., ABAQUS CAE [3], and offer a compromise by producing models that predominately consist of the preferred hex elements while providing the desirable more automatic characteristic of a tet mesher. Instead of automatically meshing with tets for the entire mesh, the automation is applied only to regions where the hex mesher has trouble. Despite the accuracy sacrificed in certain regions, the reduced meshing time for the analyst may be well worthwhile and the solution time typically is significantly less than with an all tetrahedral mesh.

Classical finite element analysis (FEA) continues to be a primary computational method of choice for most solid mechanics applications and the explicit method is significantly used by analysts in many industries such as defense, crashworthiness, and metal forming. The explicit lumped mass approach, without the use of a stiffness matrix, is well suited for rapidly changing/high rate short duration applications, but can produce distinct nuances and severely affect element performances differently than in typical static/implicit methods. Isogeometric analysis (IGA) is another recent development in FEA that is also primarily motivated by a desire for accurate meshing ease, e.g., [4]. IGA exploits underlying CAD representations (e.g., NURBS, Bézier curves, etc.) to produce highquality hexahedral meshes that are easily generated, because the elements are well-shaped in the CAD geometry. This approach can benefit from high-order elements and, as it avoids the need for element types other than hexahedrals, may vie with tetrahedral and Hex-Dominant approaches for meshing and modeling effectiveness. IGA is gaining popularity and has been extended to explicit analyses, but it is still not mature for the extremely high rate applications (with severe distortion, contact-impact, etc.) commonly encountered in explicit analysis. It too should not be expected to perform the same in static/implicit analysis as it does in explicit methods. Nevertheless, IGA appears to provide a useful capability for explicit analysis that has historically been limited to standard first-order elements in popular production software. As another alternative with contrasting characteristics to IGA, the inclusion of a comprehensive library of reliable second-order formulations would also seem to be an important addition to explicit analysis codes.

Second-order wedge formulations for static/implicit formulations have been long contained in common production software, e.g., ABAQUS [3] and TEXGAP-3D [5]. Common are the fifteen node "serendipity" wedge element, containing six vertex nodes and nine midedge nodes, and the 
eighteen node wedge element with additional nodes at the center of the three quadrilateral faces. These wedge types are compatible with twenty node "serendipity" and twenty-seven node Lagrange hexahedral elements, respectively, and both are compatible with ten node "serendipity" tetrahedrons at their triangular faces. Jog [6] presented a twenty-one node hybrid wedge element for linear static applications, which uses the same basic shape functions as will be used herein. Second-order elements can inherently and accurately represent curved shapes and model bending, particularly for thick to moderately thin beams, plates, and shells, but without using artificial hourglass control or by adding any special modes or treatments. Even higher-order elements, e.g., cubic, quartic, etc., can further reduce shear locking, but a point of diminishing returns on interpolation order exists, particularly for explicit methods where the time increment size is related to nodal spacing. While performing well as flexural elements, second-order ones still maintain their versatility as solid elements. In contrast to structural elements, they can innately model contact on multiple surfaces of the same element. With linear or higher stress/strain representations, selective reduced integration or reduced order (mixed/hybrid) pressure formulations can be used effectively to address volumetric locking on a single element basis within general unstructured meshes [3, 5]. Unfortunately, fifteen and eighteen node wedges produce negative and zero lumped vertex node masses, respectively, via row summation or nodal integration, and serendipity elements produce peculiar consistent nodal forces, which effectively eliminates both for useful explicit analysis. A uniform traction applied to an eight node quadrilateral face, for example, produces negative nodal forces at the vertices. Similarly, a uniform traction applied to a six node triangular face produces zero nodal forces at the vertices so that all of the loading is applied at the midedge nodes. Both pose a dilemma when the force is used to invoke a tensile release rule for a contacting node. Although not a problem for static/implicit methods, such nodal loadings also pose a quandary for explicit lumped mass solution methods. Unlike a static/implicit solution method that couples nodal responses via the stiffness matrix, the explicit solution is completely uncoupled for each degree of freedom so that the incremental response can be abnormal at vertex nodes. For instance, vertex nodes with zero external loads can only move by the internal resistance forces, i.e., in the next time increment, as a result of the lumped mass uncoupling.

Either the first- or the second-order elements would seem to be a reasonable compromise to general situations and thus rational to include both as options in explicit codes. Robust truly secondorder elements, however, have not been contained in explicit solid dynamics programs until recently. The expense and complexities of these elements are unattractive (as in static/implicit codes), but another reason has been skepticism in a satisfactory mass matrix diagonalization (lumping). Especially with popular reduced integration methods, second-order elements in static/implicit analyses can also be more susceptible to distortion issues than first-order ones, which is a particular concern for many explicit analyses that involve severe deformations. References $[7,8]$ presented reliable second-order hexahedrons and tetrahedrons with well-defined lumped masses and contact tractions that also perform well both with nearly incompressible materials and in flexural applications. The IMPETUS AFEA [9] explicit finite element code reports to have recently implemented second- and third-order elements, including eighteen and forty node wedge elements that are compatible with their hexahedral and tetrahedral elements, but little information is provided about their element formulations - it is not clear if mass lumping is used and if so, how they overcome the poor lumping and the problem of zero vertex forces on triangular faces, etc. Likewise, Xiang et al. [10] presented a ten node tetrahedral formulation with standard quadratic interpolation functions and all positive lumped masses, but did not explain their lumping procedure nor rigorously demonstrate its performance in an explicit method, e.g., address the zero vertex force problem with lumped masses. That is, comparisons to analytic or other computed methods were not reported, but their results at least qualitatively appear to be realistic. In any case, neither of these works appears to use the identical elements and approach described herein.

In this paper, reliable second-order wedge elements for lumped mass explicit solid dynamics, without resorting to mass scaling or other special procedures, are sought to have both reasonable computational costs and worthwhile numerical properties, particularly suitable for low interest transition and fill regions. Following the results of Danielson and O'Daniel [7] for hexahedral 
elements and the authors' more recent experiences with tetrahedrons [8], nodes at the centroids of the element and on all faces are added to the standard fifteen node wedge to produce twenty-one node formulations with the goal to have both well-defined lumped masses and surface tractions on the seven and nine node faces. Compared with the additional computations resulting from increased degrees of freedom and bandwidth/fill for equation solving in static/implicit methods, the computational increase for three to six additional nodes is small with the explicit method. The elements presented herein use standard displacement-based and shape/interpolation function approaches, typical quadrature rules, and the well-known row summation mass lumping. The twenty-one node wedge, however, is not common and particularly does not appear to be available in any existing explicit solid dynamics software. The elements are evaluated for problems involving large strains, deformations, and distortions with significant bending as well as three-dimensional applications and contact-impact, and include linear elastic and elastic-plastic material models. The elements are also evaluated for different numerical integration schemes. To fully assess the performance of the mass lumping method for transient analyses, the examples exclusively use an explicit central difference temporal integration without mass scaling or mass damping. The secondorder wedge meshes are typically more computationally intensive than corresponding all-brick element models. These elements, however, provide an important modeling alternative in explicit dynamics codes, as second-order elements have distinct advantages for certain applications, especially for flexure, curved geometries and unstructured meshes with nearly incompressible materials, and these wedge elements would thus be useful for second-order hexahedral-dominant meshing approaches.

\section{Governing equations}

The basic form of the Lagrangian nonlinear equations is derived as that in standard texts, e.g., [1114]. As shown in Fig. 2, the motion is described by the current position of any point, $\mathbf{x}$, at time, $t$, related to the undeformed position, $\mathbf{X}$, using the displacements, $\mathbf{u}$, from the undeformed configuration by

$$
\mathbf{X}=\mathbf{X}+\mathbf{u}(\mathbf{X}, t)
$$

The following virtual work statement for large strain/deformation dynamic equilibrium at any time is defined with the body forces per unit mass, b, e.g., gravity accelerations, the applied surface tractions, $\hat{\mathbf{t}}$, and the inertia forces all moving through virtual displacements, $\delta \mathbf{u}$, from the deformed configuration

$$
\int_{V_{d}} \boldsymbol{\rho}_{d} \ddot{\mathbf{u}} \cdot \delta \mathbf{u} d V_{d}=\int_{V_{d}} \boldsymbol{\rho}_{d} \mathbf{b} \cdot \delta \mathbf{u} d V_{d}+\int_{A_{d}} \hat{\mathbf{t}} \cdot \delta \mathbf{u} d A_{d}-\int_{V_{d}} \boldsymbol{\sigma}: \frac{\partial \boldsymbol{d} \mathbf{u}}{\partial \mathbf{x}} d V_{d}
$$

where $\delta$ is the variational operator designating a virtual quantity, the subscript $d$ refers to the deformed configuration, each dot refers to differentiation with respect to time, $\rho_{d}$ is the current mass density, and $A_{d}$ and $V_{d}$ are the deformed configuration surface area and volume, respectively. $\boldsymbol{\sigma}$ are Cauchy (true) stresses. The displacement boundary conditions in Eq. (2) are satisfied by explicitly controlling the motion of each appropriate nodal point. 


\section{Finite element formulation}

\subsection{Discrete equations of motion}

Eq. (2) is spatially discretized into a mesh of standard displacement-based isoparametric Galerkin elements; their general formulations can be found in texts for many different element types, e.g., [1114]. For the twenty-one node quadratic wedge formulations developed herein and depicted in Fig. 1, there are two types of edge nodes, ones adjacent to triangular/quadrilateral faces and ones adjacent only to quadrilateral faces, and two types of face nodes, triangular and quadrilateral. The edge nodes are all midedge nodes (at center), and the triangular face nodes are located at the face centroid. As in [6], the shape functions are constructed by using seven triangular planar functions, determined by evaluation on a face of a fifteen node tetrahedron [15], and one-dimensional Lagrange polynomials along the wedge axis.

Defining $q$ as a generalized degree of freedom, the general elemental equation from Eq. (2) for node, $I$, is

$$
\left[\int_{V_{o}} \rho_{o} \ddot{u}_{q} h_{I} d V_{o}\right] \delta q_{I}=\left[\int_{V_{o}} \rho_{o} b_{q} h_{I} d V_{o}+\int_{A_{d}} \hat{t}_{q} h_{I} d A_{d}-\int_{V_{d}} \boldsymbol{\sigma}: \frac{\partial h_{I}}{\partial \mathbf{x}} d V_{d}\right] \delta q_{I}
$$

where the acceleration, body force, and applied traction components with the $q$ subscript are the values in the direction of the generalized degree of freedom. The virtual quantities are virtual nodal displacements that are now factored out, so that the elemental equation for each degree of freedom is defined by the expressions within the brackets of Eq. (3). The basic finite element equations of motion for the element at time, $t$, are determined by evaluating the integrals in Eq. (3), which written in matrix form is

$$
\mathbf{M} \cdot \ddot{\mathbf{q}}^{t}=\mathbf{B}^{t}+\mathbf{P}^{t}-\mathbf{F}^{t}
$$

where $\mathbf{M}$ is the mass matrix, $\mathbf{q}$ is the generalized nodal displacement vector, $\mathbf{B}$ is the vector of body forces, and $\mathbf{P}$ is the vector of applied loads. $\mathbf{F}$ is the vector of internal resistance forces, which is determined using various linear and nonlinear measures of stress and strain (and/or their rates) along with the associated constitutive relation for each material. Large deformations are accommodated by the incremental transient procedure discussed in Section 3.4 below.

\subsection{Selective reduced integration}

Selective Reduced (SR) integration is chosen here to simply address volumetric locking for nearly incompressible materials, so that the new lumped mass elements can be evaluated for an important class of applications with the explicit method. Mixed displacement-volumetric/pressure interpolation formulations are very similar or can be equivalent to selective reduced integration, e.g., [11-14, 16], but some have also been shown to have advantages over selective reduced integration in static/implicit methods for general material models. Mixed formulations (two- or three-field) have not been commonly used in explicit dynamics software, primarily because the predominant uniform stress/strain elements do not volumetrically lock. First-order SR and B-bar type elements, however, have emerged in explicit codes, e.g., [17-19], as the constant dilation/pressure is convenient and simply computed. Selective reduced integration can be very accurate and will suffice in this present evaluation, but the results herein may inspire the development of more elaborate mixed versions.

The SR method is simple and straightforward whereby the Cauchy (true) stresses are

separated into its deviatoric components, $\boldsymbol{\sigma}^{\prime}$, and pressure, $p$, by 


$$
\boldsymbol{\sigma}=\boldsymbol{\sigma}^{\prime}-p \mathbf{I}
$$

where $\mathbf{I}$ is the identity tensor and the pressure is defined by

$$
p=-\frac{1}{3} \operatorname{trace}(\boldsymbol{\sigma})
$$

Inserting Eq. (6) into the last term in Eq. (4), the elemental internal force vector for node, $I$, is recast as

$$
\mathbf{f}_{I}^{\text {intemal }}=\int_{V_{d}} \boldsymbol{\sigma}^{\prime}:\left.\frac{\partial h_{I}}{\partial \mathbf{x}} d V_{d}\right|_{\text {Full }}-\int_{V_{d}} p \mathbf{I}:\left.\frac{\partial h_{I}}{\partial \mathbf{x}} d V_{d}\right|_{\text {Reduced }}
$$

so that different levels of integration may be applied to the deviatoric and pressure terms. A highorder (Full) quadrature rule is used on the deviatoric integrand, and a lower-order (Reduced) rule is used on the pressure one. Eq. (7) is convenient for material models that can separate deviatoric and dilation components by simply removing the pressure or deviatoric stresses from the two respective terms at the different integration points. This is effective in many cases. Severe loading and loading rates, however, are common in explicit analysis and reduced integration may not always capture the volume of a distorted element accurately.

Optionally, a slightly different formulation (designated LP) computes the second term in Eq. (7) first, so that the pressures at the "reduced" points can be used to interpolate values at the "full" higher-order integration points and added to the deviatoric stresses for more precise quadrature. Since the pressure is not truly a degree of freedom, this option is not a mixed method but rather a variant of the selective reduced integration concept. This is essentially a low-order projection of pressure in a displacement-based method. It can also provide the reduced volumetric values for computation of the deviatoric first term in Eq. (7), e.g., for pressure-dependent failure surfaces. Note that this formulation does not necessarily degenerate precisely to Eq. (7) under simple conditions. Like the pressure, the interpolation function gradients, $\partial h_{l} / \partial \mathbf{x}$, also have a lower order spatial representation in Eq. (7) than with full integration, so that the two different formulations may not be entirely virtual work-conjugate. This is not necessarily wrong, as Eq. (7) is also not virtual workconjugate to using full integration. The drawback is perhaps an increased potential to lock over the SR formulation, but the LP formulation should still be less prone to locking than with full integration. The differences between SR and LP, an element less likely to lock versus one with more accurate quadrature over the element volume for the pressure, are most likely small in many cases and the advantages of one over the other is probably dependent on the level of element distortion.

Modeling of the deviatoric and volumetric terms separately is viable for a large class of problems. For example, it is inherent to various constitutive models, such as common ones for metal plasticity or for nearly incompressible rubber hyperelasticity. The uncoupling of deviatoric and dilation responses is also frequently used to model all materials in shock physics formulations for solids, which generally use explicit temporal methods. Both components may be important, but the coupling may be insignificant in such high pressure applications. This is a characteristic that is typically used to distinguish a "hydrocode" from structural analysis codes and Eulerian gas-dynamic (hydrodynamic) programs [20]. Element computations can dominate the analysis in explicit methods, since the lumped mass equation solving is trivial. Therefore, the simple inexpensive SR and LP formulations can be effective for many explicit applications. For many materials subjected to lower rate (non-shock) wave propagation, however, the rigorous deviatoric-volumetric coupling may be important in explicit analyses. If the material has the potential to lock in such cases (nearly incompressible), either a mixed method should be used or modified gradients (B-bar/F-bar type methods) computed, for input into the constitutive model of the more general form in Eq. (4) [11-13, $14,16]$. These types of approaches may require noticeably more computations than the SR or LP 
formulations, but are applicable to general situations and can provide both a complete lower-order spatial representation and a high-order integration of the volumetric terms.

\subsection{Numerical quadrature}

The present study investigates numerical integration formulae with six, eight, twelve, eighteen, and twenty-one points. These employ a product of triangular quadrature rules and GaussLegendre rules along the wedge axis. The triangular rules use three, four, six, and seven quadrature points [21], which exactly integrate polynomial orders of two through five over a triangle, respectively. The Gauss-Legendre rules use two and three quadrature points [21], which exactly integrate polynomial orders of three and five over a line, respectively. The present elements produce integrands of order four, but if the element shape is skewed or has curvature, a rule for orders greater than four may be necessary to accurately integrate the effect of distortion. Specific rules will be defined for each analysis whereby "full" integration rule, for example, is designated as (7-3) and uses seven triangular points and three Gauss-Legendre points. Either a seven point triangular rule or a $3 \times 3$ Gauss-Legendre quadrilateral rule are used on appropriate element faces for all traction loadings.

The tendency to volumetrically lock will diminish when the number of degrees of freedom is increased relative to the number of incompressibility constraints in the model [22]. Generally, the volumetric term (pressure) is effectively represented by a lower order polynomial than that of either the displacement or strain, which is a linearly varying pressure here. A six point (3-2) quadrature rule is used herein for the selectively reduced pressure terms in Eq. (7), which provide a symmetric linear variation within the parent element geometry that is similar in its interpolation to the secondorder hybrid wedges in $[3,5]$.

\subsection{Explicit time integration procedures}

The explicit method will include additional aspects not used in static and implicit analysis software, such as mass lumping, stable time increment determination, and artificial bulk viscosity. To solve Eq. (5), explicit methods avoid iteration, e.g., Newton-Raphson, and the factorization of tangent stiffness matrices for static/implicit methods by solving it directly with a large series of much smaller time increments. To be computationally feasible, the mass matrix in Eq. (5) is typically lumped to a diagonal one making the solution trivial for each increment. As a result, elements may perform significantly differently in static/implicit methods than in explicit ones. This is particularly the case for higher-order elements. As mentioned above, however, row summation mass lumping for serendipity elements produces negative vertex values that can be detrimental for explicit methods [23]. In addition, references [7, 8] demonstrated that spurious zero energy ("hourglass") modes, which are prevented by surrounding elements when using a stiffness matrix, can propagate through a lumped model of second-order brick elements in an explicit analysis.

Using a typical explicit method, the global form of Eq. (5) for the entire mesh is first used to compute the generalized nodal accelerations, $\ddot{\mathbf{q}}^{t}$. Next, a modified central difference scheme is used for temporal integration of the velocities and displacements, e.g., [14, 24].

$$
\begin{gathered}
\dot{\mathbf{q}}^{t+\frac{\Delta t^{n+1}}{2}}=\dot{\mathbf{q}}^{t-\frac{\Delta t^{n}}{2}}+\left(\frac{\Delta t^{n+1}+\Delta t^{n}}{2}\right) \ddot{\mathbf{q}}^{t} \\
\mathbf{q}^{t+\Delta t^{n+1}}=\mathbf{q}^{t}+\Delta t^{n+1} \dot{\mathbf{q}}^{t+\frac{\Delta t^{n+1}}{2}}
\end{gathered}
$$


where $\Delta t$ with superscripts $n+1$ and $n$ refer to the current and previous time increments, respectively. Using the results from Eqs. (8-9), a new configuration is determined with Eq. (1), and updated integrals in Eq. (4) are evaluated for a new increment.

\subsubsection{Mass lumping schemes}

Since a stiffness matrix is not used in the explicit method, the only coupling among degrees of freedom in the computation of the accelerations, resulting from incremental out of balance forces, is in the mass matrix of Eq. (4). For typical explicit methods, the elemental mass is lumped at the nodes, so that the mass matrix is diagonal and the solution of the accelerations (and resulting velocity and displacement changes) is uncoupled within each increment. Mass lumping is a cornerstone of explicit methods [14, 17-19, 24]. Using Eqs. (4)-(5), the $I J$ nodal component of the consistent mass matrix is $[11-14,16]$

$$
M_{I J}=\int_{V_{o}} \rho_{o} h_{I} h_{J} d V_{o}
$$

Following [7, 8], the second-order wedge elements considered herein use a row summation scheme for mass lumping. This method sums all of the components in the row and places it on the diagonal $[11,16]$. The lumped mass matrix is then zero for non-diagonal components, such that

$$
\begin{aligned}
& M_{I I}=\sum_{J=1}^{\# \text { Nodes }} \int_{V_{o}} \rho_{o} h_{I} h_{J} d V_{o}=\int_{V_{o}} \rho_{o} h_{I} d V_{o} \\
& M_{I J}=0, I \neq J
\end{aligned}
$$

In general, row summation produces a non-uniform mass lumping. Note that the rightmost diagonal integral in Eq. (11) is a result of the shape functions all summing to unity at any point and thus producing a term that is quite similar to the body force integral in Eq. (4). This provides insight into row summation, as the mass is distributed to the nodes in the same way as a constant body force. For the twenty-one node wedge elements described herein, the (7-3) quadrature rule is used to evaluate the mass matrix integrals using Eq. (11). Comparative nodal values for the parent element of unit mass are given in Table 1 for both the fifteen node serendipity and eighteen node wedge elements. The lumping is highly non-uniform, but is identical for nodes of the same type. Note that the fifteen node element has negative lumped values for the vertex nodes. The eighteen node element has zero lumped values for both the vertex and the quad-quad midedge nodes. For the twenty-one node element, the lumping is quite non-uniform as the centroidal node has thirty percent of the total mass.

Nodal integration or "optimal" lumping [23], e.g., via Lobatto quadrature rules, naturally produce a diagonal mass matrix. As mentioned in [7], Eq. (11) and Lobatto nodal quadrature methods will produce identical masses for a mesh of perfectly shaped elements. The Lobatto rules $[11,25]$ are fine for well-shaped elements, but integrate at a lower precision than possible with Gauss-Legendre quadrature. This was shown to be problematic sometimes with irregularly shaped elements in references $[7,8]$, since the Lobatto rule order cannot be increased and still remain at the nodes for the natural diagonalization.

\subsubsection{Critical time increment}

Explicit codes frequently approximate the critical increment using only individual elemental solutions, either by rigorous eigenvalue or by simple reduced one-dimensional wave propagation 
approaches [13, 17-19, 24]. Unfortunately, a single small element included in a mesh of much larger elements dictates the increment size when the global system produces a much larger one-a situation that can be efficiently solved by subcycling $[26,27]$. Recently, however, several codes have also included methods to efficiently approximate the actual global system eigenvalue, e.g., [3, 17, 18]. These methods are continuing to evolve, as the cost savings in using a larger increment is not always worth the cost of the more intensive computation of the approximate global eigenvalue. In addition, selective mass scaling strategies have emerged [28] that can increase the critical time increment without significantly affecting specific dynamic responses. For the present study, the simple onedimensional wave propagation approach is adopted. The shortest distance between any two nodes in each element is computed, and sound wave speed of the material, $c_{d}$, is used to compute the time required to transverse that distance to approximate a critical increment, which requires a reduction factor. The authors found by trial and error that a value of generally about $1 / 2$ for well shaped elements is good for wedges and a value of 0.7 is a good choice for the Hex-Dominant meshes.

\subsubsection{Artificial bulk viscosity}

An artificial viscosity, $p_{\mathrm{abv}}$, is typically added to the pressure in explicit methods $[3,17-19,20,23$, 29 ] to prevent element collapse in the presence of high velocity gradients and to quiet truncation frequency oscillations or "ringing". This method automatically treats the shocks, and the Hugoniot jump conditions remain valid across the shock transition while the solution is unperturbed away from the shock. Ideally, the artificial viscosity would be added only to the highest mode of the element, but for computational ease, this is usually added only as a volumetric or "bulk" response using the trace of the velocity gradient (volumetric strain rate), which herein is

$$
p_{a b v}=-B_{1} \rho_{d} c_{d} l_{e} \cdot \operatorname{tr}\left(\frac{\partial \dot{\mathbf{u}}}{\partial \mathbf{x}}\right)+B_{2} \rho_{d}\left[l_{e} \cdot \operatorname{tr}\left(\frac{\partial \dot{\mathbf{u}}}{\partial \mathbf{x}}\right)\right]^{2}
$$

$\mathrm{B}_{1}$ and $\mathrm{B}_{2}$ are input parameters with default values of 0.06 and 1.5 for the linear and quadratic terms, respectively, and which approximates the characteristic elemental length, $l_{e}, p_{\mathrm{abv}}$ is added to the pressure at all integration points in all elements, except for SR type elements (WEG21SR, WEG21LP6, etc.) where it is only added to the points for the deviatoric quantities. This rule was determined by numerical experimentation, and using full integration on a pressure for an incompressible material may seem counter-intuitive. The artificial viscosity, however, is intended for all modes, and experience so far has shown this to work the best.

\subsection{Element types}

For this study, Table 2 describes the element nodal interpolation and integration types for the terms in Eq. (4). Note that a complete element formulation also includes a mass lumping scheme for the term on the left hand side of Eq. (4). Row summation lumping is used as the default in all (secondorder element) analyses unless otherwise specified. The first six element types listed in Table 2 are used herein for comparison purposes, and the second-order ones also are used in Hex-Dominant models and do not possess any artificial hourglass control, incompatible modes, or other special bending treatments. The WEG21 and WEG21R elements are intended for compressible materials and use twenty-one ("Full") and eighteen ("Reduced") quadrature points, respectively, unless otherwise noted for the Reduced quadrature. To evaluate the explicit scheme for nearly incompressible material models, the WEG21SR/WEG21RSR selective reduced integration and WEG21LP6/WEG21RLP linear pressure elements, which use twenty-one/eighteen quadrature points on the deviatoric terms and six pressure points, should be less susceptible to volumetric locking. 
These full and selective reduced integration elements may still shear lock in thin bending applications, but their applicability also has a practical limit, as a consequence of the associated increasingly small time increment for explicit methods.

\section{Evaluation example problems}

The above element formulations were implemented into the authors' parallel finite element code, ParaAble [30, 31], and executed for the following suite of example problems. The examples vary in their use of English, SI, and non-dimensional units, so as to be consistent with the analyses in the literature that are used for comparison. Analysts may directly define wedges in non-critical fill regions, but automatic Hex-Dominant meshers, however, may not always easily fill with wedges only in areas of little interest. The below examples will first evaluate the second-order wedge elements on their own, then place wedge elements in critical areas of a Hex-Dominant mesh so as to evaluate their performance in a nearly worst case situation, and then the final example demonstrates where their judicious placement provides significant advantages for Hex-Dominant modeling using a combination of wedges, hexahedra, and tetrahedra.

\subsection{Large deformation isotropic beam vibration}

This example models the large deformation vibration of a simply supported linear elastic beam subjected to instantaneous continuous upward uniform loading on its top. The problem corresponds to one defined in [8, 32-34]. The beam uses non-dimensional units with a modulus of elasticity of $1.0 \times 10^{9}$, Poisson's ratio of 0.0 , mass density of 1000.0 , and dimensions of $0.8 \times 0.1 \times 0.025$. As depicted in Figs. 3 and 4, the WEG21 elements can be oriented in three different ways. The three models use arrangements of two wedge elements within each of four hexahedral volumes along the length for a total of eight elements. A single hexahedral volume is used through the beam thickness and in the out-of-plane $\mathrm{X}_{3}$ direction. The example can be challenging for continuum elements, as the localized simple support at a point significantly stimulates hourglass modes. For the simple support, the midsurface nodes are pinned at the left end of the mesh, and only half the beam is modeled using symmetry. In contrast to [8,32-34], motion herein is not restrained in the $\mathrm{X}_{3}$ direction, except at the pinned support, so as to make the problem even more difficult for solid elements, i.e., eliminate the potential additional stability of the $\mathrm{X}_{3}$ restraints.

Fig. 3 also shows the deformed Mesh-1. The corresponding predicted midsurface deflections for all three models, given in Table 3, are in agreement with the published predictions and the Mesh1 time histories shown in Fig. 5 compare well with the HEX27 model prediction. As also shown in Fig. 5, the WEG21 solutions for all three nodes in the out-of-plane $\mathrm{X}_{3}$ direction showed close but slightly different results, because of the mesh skew symmetry. To investigate mesh orientation effects, the two different models in Fig. 4, were used and produced the very similar results given in Table 3. The analysis was also performed with the WEG21R model using eight (4-2), several twelve (4-3, 6-2), and eighteen (6-3) point quadrature rules. All WEG21R analyses demonstrated spurious hourglass deformations as the deformation becomes large except for the default eighteen (6-3) point rule, which produced nearly identical results to the WEG21 analysis (see Table 3).

\subsection{Large deformation elastic and elastic-plastic cantilever vibration}

This application predicts large deformation vibration of a cantilevered beam modeled both as linear elastic and as elastic-plastic with isotropic hardening. Again, the model uses non-dimensional units with the problem originally posed in $[35,36]$. Its beam dimensions are $25 \times 4.0 \times 1.0$ with mass density of 1.0 , modulus of elasticity of $1.0 \times 10^{4}$, Poisson's ratio of 0.25 , and for the elastic-plastic material, yield stress of 300 and plastic modulus of 100. It is loaded by a tip shear stress that varies 
in the vertical $\mathrm{X}_{2}(\mathrm{y})$ direction by $t_{\mathrm{y}}=15\left(1-\mathrm{y}^{2} / 4\right)$. These examples are challenging for lower-order elements, as the variation of the shear stress load through the cantilevered beam thickness is quadratic. As depicted in Fig. 6, combinations of wedge and brick elements are used in each mesh of the beam. Two wedge elements are used at the wall in hexahedral arrangements that are connected to three more brick elements along the length for a total of five elements. Figure 6 shows the two possible arrangements of the wedges elements, Mesh-A and Mesh-B, that can connect to these three brick elements. A single element layer is used through the beam thickness and in the out-of-plane $\mathrm{X}_{3}$ direction. Motion is restrained to be only in the $X_{1}-X_{2}$ plane. The entire left edge of the mesh is restrained for the fixed support of the cantilevered beam.

Belytschko and Bindeman $[35,36]$ demonstrated that flexural performance of various perturbation and assumed strain hourglass control methods varied significantly for elastic and elasticplastic materials. For these two cases, the accurate level of the perturbation hourglass control stiffness parameter was significantly different, and all the methods they considered had a slow convergence rate for the elastic-plastic case. Figure 7 shows the predictions from Mesh-A and Mesh B using WEG21R elements for elastic and WEG21RLP for elastic-plastic analyses along with ones using HEX27 and HEX27SR elements for comparison. The time histories are slightly more flexible than the first-order element predictions given in $[35,36]$ for both elastic and a very fine mesh $(32 \times 192)$ of two-dimensional elastic-plastic elements. In $[8,36]$, the predictions of several different first-order brick types appear to be similarly converging close to the HEX27SR predictions in Fig. 7. As seen in Fig. 7 and Table 4, the model predictions using the library of WEG21 type elements are generally very close to the all HEX27 model elastic predictions. The predictions for the elasticplastic case, however, are somewhat stiff, but still reasonably accurate and similar to the coarse mesh first-order element predictions in $[35,36]$. Note that the WEG21RLP element demonstrates the most consistently accurate prediction for all cases.

\subsection{Cylindrical bar impact}

This example is a cylindrical bar impacting a rigid wall at $227 \mathrm{~m} / \mathrm{s}$. The model uses a quarter symmetric mesh and definition similar to that in [3, 37-39], except that here, rigid wall frictionless contact is specified instead of a symmetry plane. The cylinder face impacting the wall actually lifts off in several places, so the contact specification here is more precise and complex. Nevertheless, the predicted final bar dimensions using either wall treatment are quite similar. The bar is $3.24 \times 10^{-2}$ $\mathrm{m}$ long with a $3.2 \times 10^{-3} \mathrm{~m}$ radius. The copper isotropic hardening plasticity model uses a modulus of elasticity of $1.17 \times 10^{11} \mathrm{~Pa}$, Poisson's ratio of 0.33 , yield stress of $4.00 \times 10^{8} \mathrm{~Pa}$, tangent plastic modulus of $1.00 \times 10^{8} \mathrm{~Pa}$, and mass density of $8930 \mathrm{~kg} / \mathrm{m}^{3}$. Figure 8 shows two different undeformed models with the same number of elements, one using only HEX27SR elements and a hexahedraldominant one using wedge elements near the axis of rotation with HEX27SR elements placed elsewhere.

Figure 9 shows the final predictions of the all HEX27SR model and the hexahedral-dominant one using both WEG21SR and WEG21LP6 elements. All three predictions in Fig. 9 compare favorably with the results in [3, 37-38] and the analytical and experimental predictions of Wilkins and Guinan [39]. Analyses were also performed using WEG21RSR and WEG21RLP elements, which used slightly less CPU time, but produced very similar predictions to their fully integrated counterparts. Although it is easier to mesh the axisymmetric object with the aid of wedge elements, this specific problem can be easily meshed and efficiently solved using all hexahedral elements. The all HEX27SR model predicts a slightly less axisymmetric response, but it has larger time increment and thus uses about $40 \%$ less CPU time than the hexahedral-dominant models. Nevertheless, the example does demonstrate that the twenty-one node wedge element can accurately model the high distortion of such applications with an explicit method as part of an unstructured hexahedraldominant mesh. 


\subsection{Impact of a stiffened cylinder into a rail}

This example, which appears in the DYNA3D Example Problems Manual [38], uses a quarter symmetric mesh of a stiffened cylinder impacting a rigid $1.5 \mathrm{in}$. wide rail at $660 \mathrm{in} / \mathrm{s}$ (see Fig. 10). The $12.0 \mathrm{in}$. long cylinder is $1 / 4 \mathrm{in}$. thick with a $9.0 \mathrm{in}$. diameter. The elastic perfectly plastic material model of the cylinder uses a modulus of elasticity of $30.0 \times 10^{6}$ psi, Poisson's ratio of 0.3 , tangent plastic modulus of 0.0 , yield stress of $1.9 \times 10^{5} \mathrm{psi}$, and mass density of $7.346 \times 10^{-4} \mathrm{lbf}-\mathrm{s}^{2} / \mathrm{in}^{4}$. The dense stiffener material model is linear elastic with a modulus of elasticity of $60.0 \times 10^{6}$ psi, Poisson's ratio of 0.3 , and mass density of $1.47333 \times 10^{-2} \mathrm{lbf}-\mathrm{s}^{2} / \mathrm{in}^{4}$. The contact is assumed to be frictionless. Four different meshes, shown in Fig. 10, are used: (a) a baseline model with all hexahedral elements [8], (b) a hexahedral-dominant one using WEG21LP6 elements in the impact zone and bricks everywhere else, (c) a mesh with all of the bricks of the baseline model converted to six tetrahedral (TET15LP4) elements, and (d) an unstructured tetrahedral (TET15LP4) mesh with all nearly uniform and well shaped elements restricted to a single element layer through the thickness of the thinner cylinder. Full integration elements (i.e., HEX27, WEG21, TET15) are used for the stiffener elements in all four models.

As shown in Fig. 11, the cylinder impacts the rail and rebounds from it with a permanent plastic dent. Figure 12 shows the time history of the maximum indentation for the different models, computed by subtracting the free-end displacement from that at the center of the impact. Except for Model-c, the models vibrate about a slightly more flexible indentation than the permanent test value. Again, this problem can be easily meshed and efficiently solved using all hexahedral elements, but it demonstrates that the twenty-one node wedge elements can accurately model the large deformation elastic-plastic flexural impact model with an explicit method, which required only about $7 \%$ more CPU time than the baseline Model-a. The Model-b predictions are slightly stiffer than the baseline predictions, but are especially good considering that the wedge elements, as part of a hexahedraldominant mesh, would not typically be intended to compose the most critical part of the model. The unstructured tetrahedral Model-d predictions lay almost on top of the Model-a baseline history in Fig. 12, but Model-d required over 500 times the CPU time of Model-b. The tet meshing might be more efficient by using a bias near the impact region, but even so, Model-c without such additional bias refinement still required nearly twenty times the CPU time of the hexahedral-dominant Model-b.

\subsection{Penetration into concrete using cavity expansion functions}

This example models the penetration of a solid ogive-nose projectile into a concrete half-space using cavity expansion penetration resistance functions. The material properties are the same as in the cavity expansion resistance function example in the PRONTO 3D manual [40] and attributed to Warren and Tabbara [41] with penetration test data from Frew, et al. [42]. The projectile has a 3.0 CRH ogive-nose, shank length of $169.5 \mathrm{~mm}$, nose length of $33.7 \mathrm{~mm}$, and shank diameter of 20.3 $\mathrm{mm}$. It is made of $4340 \mathrm{R}_{\mathrm{c}} 45$ steel and modeled with a power law hardening elastic-plastic model with modulus of elasticity of $206.8 \mathrm{GPa}$, Poisson Ratio of 0.32 , yield stress of $1.207 \mathrm{GPa}$, hardening constant of $382 \mathrm{MPa}$, hardening exponent of 0.266 , plastic modulus of $141 \mathrm{MPa}$, and mass density of $7810 \mathrm{~kg} / \mathrm{m}^{3}$. The concrete has an unconfined strength, $\mathrm{f}_{c}$, of $58.4 \mathrm{MPa}$, mass density of $2320 \mathrm{~kg} / \mathrm{m}^{3}$, and an $\mathrm{S}$ factor for the cavity expansion function of 9.037. A Hex-Dominant model is easily created from a second-order two-dimensional mesh that is generated by a simple paving approach on the half cross-section, which typically requires very little user intervention. That mesh is then simply revolved about the projectile axis to generate second-order hexahedrals away from the axis and wedge elements along the axis from the quadrilaterals, and tetrahedral elements on the axis at the nose from the triangle. The resulting model, shown in Fig. 13, has two tetrahedra, twenty-eight wedge elements, and twenty-six hexahedra with a total of 591 nodes. If the two-dimensional mesh had triangles off the axis, these would become wedges, and a triangle with a single vertex on the axis would become a pyramid by this rotation - all of which would comprise a fully compatible HexDominant mesh. 
As seen in Fig. 14, the half-symmetry model predictions are in good agreement with the normal impact predictions and test data of the examples in [40, 42]. This model can also predict many other impact conditions by either a non-normal velocity vector or rotation of the mesh within the plane. Although only two elements are used around the half-circumference, there are four second-order elements through the thickness of the projectile, which by the results of Examples 4.1, 4.2, and 4.4, indicate that it might also reasonably predict flexure responses. In Fig. 15, the final deformed model plots, for an impact velocity of $500 \mathrm{~m} / \mathrm{s}$ with an Angle of Attack (AOA ) of 5.0 degrees (velocity vector relative to projectile axis), are shown using the mesh of reference [40] and that in Fig.13. The projectile models both predict very similar final positions at about a $0.27 \mathrm{~m}$ depth and a double permanent bending in the aft end. The first-order model used default values for the hourglass control and the second-order model of course models hourglass model naturally without resorting to artificial control. Although the model in Fig. 13 has about $28 \%$ more quadrature points (1808 for half-symmetry) than the number of first-order hexahedra (2816 for full-symmetry) in [40], the larger elements in the Hex-Dominant model produces a time increment of more than twice that of first-order model. A typical cpu time for the full-symmetry first-order model (with ParaAble) is about 3.5 times more than for the half-symmetry Hex-Dominant model.

\section{Performance assessment and comments}

Examination of the results from the examples in the previous section yields the following important observations.

- Twenty-one node wedge elements using row summation mass lumping performed satisfactorily in all cases including highly nonlinear applications with bending, nearly incompressible materials, contact-impact, and severe distortions.

- The "full" twenty-one (7-3) point quadrature rules, i.e., for WEG21, WEG21SR, WEG21LP6, and the slight reduction over the triangular cross-section eighteen (6-3) point quadrature rule, i.e., for WEG21R, WEG21RSR, WEG21RLP, were found to be important. The other reduced integration rules produced inaccuracies associated with large distortions and spurious zero energy (hourglass) modes.

- The accuracy of the wedge elements were only slightly less than that of brick elements and along with their efficiency to transition and fill specific volume types makes them attractive for hexahedral-dominant meshing that were shown to be an effective alternative to alltetrahedral meshing.

The primary finding was the robust accuracy of the row summation lumping for the twentyone node wedge element in contrast to the unsatisfactory mass lumping of the fifteen and eighteen node ones, making it a good performing transition element for hexahedral-dominant meshing with explicit time integration. The authors believe that the transient methodologies presented herein generally rival or surpass the accuracy and robustness of that for first-order wedges, and that the relative effectiveness depends on the appropriate mesh density and selection of the element type for a specific application. With the proper time increment and quadrature rule, the twenty-one node element also appears to generally perform at least as accurately in explicit methods as the fifteen and eighteen node wedges do in static/implicit analysis for nonlinear solid mechanics applications. As with the second-order hexahedron and tetrahedron [7, 8], the key was the combined inclusion of the centroidal and face nodes along with nearly full integration. High-order quadrature rules are necessary to avoid spurious zero energy (hourglass) modes that are not resisted by the diagonal mass matrix of the explicit method. The diagonalization is a benefit for modeling wave discontinuities, but also permits these spurious modes to propagate through the mesh in the same way. The stiffnesses of adjacent elements in a non-diagonal matrix, however, can suppress such modes and thus permit lower quadrature rules in unstabilized static/implicit methods. To avoid the need for a 
stabilization method, either the twenty-one (7-3) or the eighteen (6-3) point quadrature rule can be applied for the standard element and for the deviatoric terms of the selective reduced integration elements in an explicit method.

The slight reduction over the triangular cross-section of the (6-3) eighteen point quadrature rule has been accurate without any numerical issues for all analyses that the authors have performed. The mixture of quadrature levels, exact for a fourth-order polynomial over the triangle and for a fifth-order one along the length, may seem unsettling, but the wedge element interpolation is also directional. In comparison with the (7-3) rule, the authors have found that the (6-3) rule is as accurate or slightly better and requires about $10 \%$ less CPU time (per element). The authors still recommend the full (7-3) rule for the mass matrices, which would also require computing the shape functions and their derivatives at a second set of points when the (6-3) rule is used.

The increased computations for three or six additional nodes are small with the explicit method compared to the increase associated with equation solving in static/implicit methods. The number of quadrature points in the second-order elements, however, is computationally intensive and the critical time increment can be small. The examples show that rather coarse meshes can sometimes be used with twenty-one node wedges, whereby only a single layer of elements can adequately model moderately thin structural components, which makes them more computationally competitive in flexure. As a general rule for non-flexure dominated applications, the authors' experiences are that the second-order wedge models are noticeably more expensive than models using first-order hexahedra. Unlike first-order ones, however, the second-order wedge elements, can be curved, can model flexure with a single element, and can stand on their own in an unstructured (e.g., hexahedral dominant) mesh without severe volumetric locking. The use of twenty-one node wedges in critical regions is also slightly less accurate than using second-order hexahedra, and a reduced critical time increment may affect computational time. Analysts using wedges, however, would typically expect these effects and accept the cost increase in favor of using more simplified hexahedral-dominant meshing procedures.

The present study was primarily intended to evaluate the numerical quadrature and mass lumping schemes of second-order wedge elements for explicit temporal integration methods in hexahedral-dominant models. The other numerical procedures are reasonably accurate for this assessment, but further improvements to the computational procedures might be attained. The elements are also reliable performers, but they essentially use basic straightforward displacementbased methods. Two- or three-field tri-linear pressure, hybrid, B-bar, or other typical methods would also be expected to perform reasonably well. The inclusion of contact in two of the examples was useful in the present evaluation, but it was also not very complicated. An effective method for more complex interactions that are frequent in many explicit analyses, e.g., crushing, self-contact, fracturing and new surface definition, searching, etc., has yet to be demonstrated with the curved surfaces of the twenty-one node wedges for high rate applications, but the elements appear to be robust.

The authors do not object to first-order elements with assumed strain, incompatible modes, under-integration stabilization methods, etc. The drawbacks of using these techniques certainly outweigh the problems of potential shear locking, and these element formulations possess the fundamental property of convergence, i.e., inter-element incompatibilities or hourglass modes diminish with mesh refinement. After all, mass lumping, artificial viscosity, etc. are also procedures used solely for numerical reasons. In addition, the relative amount of energy associated with controlling spurious zero energy modes is typically less in second-order elements than with firstorder elements, and thus a second-order wedge with fewer integration points and employing stabilization or other methods might be worthwhile. Nevertheless, the natural modeling of flexural modes by these second-order displacement-based elements is a highly attractive trait, especially in inelastic flexure where parameter selection, e.g. for hourglass control, is not always easy. This robustness, however, may result in a noticeable increase in computational cost. Thus, this natural modeling characteristic alone may not be a sufficient reason to use these elements in many cases. The primary advantage of the twenty-one node wedge elements presented herein is the utilization in more simplified hexahedral-dominant meshing, particularly in applications with significant bending 
and/or curvature, which may be well worth any additional computational cost that generally would be much less than with all-tetrahedral meshing.

\section{Concluding remarks}

Second-order "serendipity" wedge elements are common in static and implicit dynamics finite element codes for linear and nonlinear solid mechanics. They can perform better than first-order elements in many circumstances, particularly for modeling curved shapes and bending without artificial hourglass control, assumed strains, or incompatible modes. They can also perform well with nearly incompressible materials in unstructured meshes so as to ease mesh generation. Nevertheless, second-order wedge elements are not contained in typical explicit solid dynamics programs due to the lack of a satisfactory mass lumping and consistent nodal loading for the "serendipity" versions. Standard row summation lumping produces negative and zero vertex node masses for the popular fifteen and eighteen node wedges, respectively. Subjected to a constant pressure, the six and eight node "serendipity" faces also place zero and negative loading on vertex nodes, respectively. In this paper, twenty-one node second-order formulations, ones for compressible and ones for nearly incompressible materials, were presented and evaluated. Row summation mass lumping for the twenty-one node wedge produces non-uniform, but all positive, nodal masses. The nodal loads resulting from a uniform traction applied to both types of faces of the wedge element are also all positive. Performance was assessed in standard benchmark problems and practical applications using various elastic and elastic-plastic material models and involving very large strains/deformations, severe distortions, and contact-impact. Comparisons were also made with several other first- and second-order element formulations. The offered elements performed satisfactorily in all examples. As recently found for second-order hexahedral and tetrahedral elements, it was shown that the inclusion of face and centroidal nodes is vital for robust performance with row summation lumping. High-order "full" or slightly reduced quadrature rules were also shown to be crucial with second-order wedge elements in explicit methods so as to avoid needing stabilization/hourglass control methods. These second-order wedge elements were shown to be viable for practical applications, especially using today's parallel computers. As expected, the performances of the wedge elements were less than that of their brick counterparts, but they were still quite good, particularly if used in regions of low interest as part of a hexahedral-dominant mesh. Whereas the reliable performance is frequently attained at additional computational expense compared with first-order and brick types, these elements can be computationally competitive in flexure and have the desirable trait that they are amenable to user-defined or automatic hexahedraldominant meshing/remeshing.

\section{Acknowledgements}

Permission to publish was granted by Director, Geotechnical and Structures Laboratory. The work was supported in part by grants of computer time from the DOD High Performance Computing Modernization Program at the ERDC DOD Supercomputing Resource Center (DSRC).

\section{References}

[1] Meshkat S, Talmor D (2000) Generating a mixed mesh of hexahedra, pentahedra and tetrahedra from an underlying tetrahedral mesh. Int J Numer Meth Eng 49:17-30

[2] Owen SJ, Saigal S (2000) H-Morph: an indirect approach to advancing front hex meshing. Int J Numer Meth Eng 49:289-312

[3] Abaqus 6.12 (2012) CAE, Theory, Benchmarks, and Examples Manuals. Dassault Systèmes Simulia Corp., Providence, RI, USA 
[4] Hughes TJR, Cottrell JA, Bazilevs Y (2005) Isogeometric analysis: CAD, finite elements, NURBS, exact geometry, and mesh refinement. Comput Meth Appl Mech Eng 194:41354195

[5] Becker EB, Dunham RS (1977) TEXGAP-3D, A User Oriented Three-Dimensional Static Linear Elastic Stress Analysis Program. The Texas Institute for Computational Mechanics, The University of Texas at Austin

[6] Jog CS (2005) A 27-node hybrid brick and a 21-node hybrid wedge element for structural analysis. Finite Elements Anal Des 41:1209-1232. doi:10.1016/j.finel.2004.11.007

[7] Danielson KT, O’Daniel JL (2011) Reliable second-order hexahedral elements for explicit methods in nonlinear solid dynamics. Int J Numer Meth Eng 85(9):1073-1102. doi: $10.1002 / \mathrm{nme} .3003$

[8] Danielson, KT (2014) Fifteen Node Tetrahedral Elements for Explicit Methods in Nonlinear Solid Dynamics. Comput Meth Appl Mech Eng 272:160-180, ISSN 0045-7825, http://dx.doi.org/10.1016/j.cma.2014.01.012

[9] IMPETUS AFEA (2012) SOLVER, User Documentation, version 3.0 beta, IMPETUS AFEA, Flekkefjord, Norway

[10] Xiang J, Munjiza A, Latham J-P (2011) Finite strain, finite rotation quadratic tetrahedral element for the combined finite-discrete element method. Int J Numer Meth Eng 79(9):946978. doi: 10.1002/nme.2599

[11] Zienkiewicz OC, Taylor RL, Zhu JZ (2005) The finite element method: its basis and fundamentals, sixth edition. Elsevier Butterworth-Heinemann, Burlington, MA

[12] Zienkiewicz OC, Taylor RL (2005) The finite element method for solid and structural mechanics, sixth edition. Elsevier Butterworth-Heinemann, Burlington, MA

[13] Bathe KJ (1996) Finite Element Procedures. Prentice-Hall, Englewood Cliffs, New Jersey

[14] Belytschko T, Liu WK, Moran B (2000) Nonlinear Finite Elements for Continua and Structures. John Wiley \& Sons, New York

[15] Bertrand FH, Gadbois MR, Tanguy PA (1992) Tetrahedral elements for fluid flow. Int J Numer Meth Eng 33(6):1251-1267

[16] Hughes TJR (1987) The finite element method linear static and dynamic finite element analysis. Prentice-Hall, Englewood Cliffs, NJ

[17] Presto User's Guide Version 4.16 (2012) Report SAND2010-3112, Sandia National Laboratories, Albuquerque, NM

[18] LS-DYNA (2012) KEYWORD USER'S MANUAL VOLUME I, Version 971 R6.1.0. Livermore Software Technology Corporation, Livermore, CA

[19] Zywicz E, Lin JI (2012) DYNA3D: A nonlinear, explicit, three-dimensional finite element code for solid and structural mechanics: version 12.1. Lawrence Livermore National Laboratory Report, LLNL-SM-599533. Livermore, CA

[20] Benson DJ (1992) Computational methods in Lagrangian and Eulerian hydrocodes. Comput Meth Appl Mech Eng 99:235-394

[21] Cowper,G R (1973) Gaussian quadrature formulas for triangles. Int J Numer Meth Eng 7(3):405-408. doi:10.1002/nme.1620070316

[22] Nagtegaal JC, Parks DM, Rice JR, (1977) On numerically accurate finite element solutions in the fully plastic range. Comput Meth Appl Mech Eng 4:153-177

[23] Malkus DS, Plesha ME (1986) Zero and negative masses in finite element vibration and transient analysis. Comput Meth Appl Mech Eng 59(3):281-306

[24] Taylor LM, Flanagan DP (1989) PRONTO 3D: A three-dimensional transient solid dynamics program. Report SAND 87-1912, Sandia National Laboratories, Albuquerque, NM

[25] Bessems D (2003) Development of an extended quadratic tetrahedron for finite element analysis of Navier-Stokes problems. MSc Thesis, Department of Mechanical Engineering, Eindhoven University of Technology, Eindhoven, Netherlands

[26] Belytschko T, Yen HJ, Mullen R (1979) Mixed methods in time integration. Comput Meth Appl Mech Eng 17/18:259-275 
[27] Neal MO, Belytschko T (1989) Explicit-explicit subcycling with non-integer time step ratios for structural dynamic systems. Comput Struct 31(6):871-880

[28] Olovsson L, Unosson M, Simonsson K (2004) Selective mass scaling for thin walled structures modeled with tri-linear solid elements. Comput Mech 34:134-136. doi: 10.1007/s00466-004-0560-6

[29] Johnson GR, Beissel SR, Gerlach CA, Holmquist TJ, Stryk RA (2010) User Instructions for the 2010 Version of the EPIC Code, Southwest Research Institute, Final Report, Contract W56HZV-06-C-0194 with U.S. Army RDECOM-TARDEC.

[30] Danielson KT, Namburu RR (1998) Nonlinear dynamic finite element analysis on parallel computers using FORTRAN 90 and MPI. Adv Eng Software; 29(3-6):179-186

[31] Danielson KT, Akers SA, O’Daniel JL, Adley MD, Garner SB (2008) Large-scale parallel computation methodologies for highly nonlinear concrete and soil applications. J Comput Civ Eng; 22(2):140-146

[32] Flanagan DP, Belytschko T (1981) A uniform strain hexahedron and quadrilateral with orthogonal hourglass control. Int J Numer Meth Eng 17:679-706.

[33] Belytschko T (1983) Correction of article by D.P. Flanagan and T. Belytschko. Int J Numer Meth Eng 19:467-468

[34] Belytschko T, Ong JS-J Liu WK, Kennedy JM (1984) Hourglass control in linear and nonlinear problems. Comput Meth Appl Mech Eng 443:251-276

[35] Belytschko T, Bindeman LP (1991) Assumed strain stabilization of the 4-node quadrilateral with 1-point quadrature for nonlinear problems. Comput Meth Appl Mech Eng 88:311-340

[36] Belytschko T, Bindeman LP (1993) Assumed strain stabilization of the eight node hexahedral element. Comput Meth Appl Mech Eng 105:225-260

[37] Zienkiewicz OC, Rojek J, Taylor RL, Pastor M (1998) Triangles and tetrahedra in explicit dynamic codes for solids. Int J Numer Meth Eng 43:565-583

[38] Lovejoy SC, Whirley RG (1990) DYNA3D example problem manual. Lawrence Livermore National Laboratory Report, UCRL-MA-105259

[39] Wilkins ML, Guinan MW (1973) Impact of cylinders on a rigid boundary. J Appl Phys 44:1200-1206. doi:10.1063/1.1662328

[40] Attaway, S. W., Brown, K. H., Mello, F.J., Heinstein, M. W., Swegle, J. W., Ratner, J. A., and Zadoks, R. I. (1998) PRONTO 3D Users' Instructions: A transient dynamics code for nonlinear structural analysis. Report SAND98-1361, Sandia National Laboratories, Albuquerque, NM

[41] Warren, T.L. and Tabbara, M.R. (1997) Spherical cavity-expansion forcing function in PRONTO 3D for applications to penetration problems. SAND97-1174, Sandia National Laboratories, Albuquerque, NM.

[42] Frew, D.J., Hanchak, S.J., Green, M.L., and Forrestal, M.J. (1998) Penetration of concrete targets with ogive-nose steel rods. Int J Impact Engng 21(6)489-497 
Table 1. Nodal values of row summation unit mass lumping for the parent wedge element; numerical values in parentheses

\begin{tabular}{|c|c|c|c|}
\hline Node Type & $\underline{\mathbf{1 5} \text { Nodes }}$ & $\underline{\mathbf{1 8} \text { Nodes }}$ & $\underline{\text { 21 Nodes }}$ \\
\hline Vertex & $-1 / 9(-0.111111)$ & 0.0 & $5 / 600(0.008333)$ \\
\hline Midedge (Tri-Quad) & $1 / 6(0.166667)$ & $5 / 90(0.055556)$ & $2 / 90(0.022222)$ \\
\hline Midedge (Quad-Quad) & $2 / 9(0.222222)$ & 0.0 & $1 / 30(0.033333)$ \\
\hline Triangular Face & N/A & N/A & $3 / 40(0.075000)$ \\
\hline Quadrilateral Face & N/A & $2 / 9(0.222222)$ & $8 / 90(0.088889)$ \\
\hline Centroid & N/A & N/A & $3 / 10(0.300000)$ \\
\hline
\end{tabular}


Table 2. Designation and description of element interpolation types and integration schemes compared in this paper

\begin{tabular}{|c|c|}
\hline HEX8FB & $\begin{array}{l}\text { 8-Node Uniform Strain Hexahedron of Flanagan-Belytschko [32] with } \\
\text { Perturbation Stiffness Hourglass Control. }\end{array}$ \\
\hline HEX27 & 27-Node Hexahedron with “Full” 3×3×3 Gauss Quadrature. \\
\hline HEX27SR & $\begin{array}{l}\text { 27-Node Hexahedron with Selectively Reduced Gauss Quadrature using } \\
3 \times 3 \times 3 \text { Integration on Deviatoric Quantities and } 2 \times 2 \times 2 \text { Integration on } \\
\text { Volumetric Terms (Pressure). }\end{array}$ \\
\hline HEX27LP8 & $\begin{array}{l}\text { 27-Node Linear Pressure Hexahedron using } 3 \times 3 \times 3 \text { Integration on } \\
\text { Deviatoric Quantities with Pressure computed at } 2 \times 2 \times 2 \text { Integration } \\
\text { Points and Interpolated for the } 27 \text { Points. }\end{array}$ \\
\hline TET15 & 15-Node Linear Pressure Tetrahedron using "Full" 15 Point Integration. \\
\hline TET15LP4 & $\begin{array}{l}\text { 15-Node Linear Pressure Tetrahedron using } 15 \text { Point Integration on } \\
\text { Deviatoric Quantities with Pressure computed at } 4 \text { Integration Points } \\
\text { and Interpolated for the } 15 \text { Points. }\end{array}$ \\
\hline WEG21 & 21-Node Wedge; Uses “Full” (7-3) Point Quadrature. \\
\hline WEG21R & $\begin{array}{l}\text { 21-Node Wedge; Uses “reduced" (6-3) Point Quadrature, Unless } \\
\text { Otherwise Noted. }\end{array}$ \\
\hline WEG21SR & $\begin{array}{l}\text { 21-Node Wedge with Selectively Reduced Gauss Quadrature using (7- } \\
\text { 3) Integration on Deviatoric Quantities and (3-2) Integration on } \\
\text { Volumetric Terms (Pressure). }\end{array}$ \\
\hline WEG21RSR & $\begin{array}{l}\text { 21-Node Wedge with Reduced/Selectively Reduced Gauss Quadrature } \\
\text { using (6-3) Integration on Deviatoric Quantities and (3-2) Integration on } \\
\text { Volumetric Terms (Pressure). }\end{array}$ \\
\hline WEG21LP6 & $\begin{array}{l}\text { 21-Node Linear Pressure Wedge using (7-3) Integration on Deviatoric } \\
\text { Quantities with Pressure computed at the (3-2) Integration Points and } \\
\text { Interpolated for the } 21 \text { Points. }\end{array}$ \\
\hline WEG21RLP & $\begin{array}{l}\text { 21-Node Reduced/Linear Pressure Wedge using (6-3) Integration on } \\
\text { Deviatoric Quantities with Pressure computed at the (3-2) Integration } \\
\text { Points and Interpolated for the } 18 \text { Points. }\end{array}$ \\
\hline
\end{tabular}


Table 3. Peak midsurface deflections for uniformly loaded simply supported elastic beam with different traction magnitudes and compared to HEX27 and reference predictions with varying hourglass stiffness coefficients (value of $\varepsilon$ in parentheses below) from Belytschko et al. [34]

\begin{tabular}{|c|c|c|c|c|c|c|c|c|c|}
\hline Traction & HEX27 & $\begin{array}{l}\text { WEG21 } \\
\text { Mesh-1 }\end{array}$ & $\begin{array}{l}\underline{\text { WEG21 }} \\
\underline{\text { Mesh-2 }}\end{array}$ & $\begin{array}{l}\text { WEG21 } \\
\text { Mesh-3 }\end{array}$ & $\begin{array}{l}\underline{\text { WEG21R }} \\
\underline{\text { Mesh-3 }}\end{array}$ & HEX8FB $^{*}$ & HEX8FB $^{*}$ & HEX8FB $^{*}$ & HEX8FB $^{*}$ \\
\hline $7.2 \times 10^{3}$ & $9.49 \times 10^{-4}$ & $9.50 \times 10^{-4}$ & $9.48 \times 10^{-4}$ & $9.49 \times 10^{-4}$ & $9.49 \times 10^{-4}$ & $\begin{array}{l}9.98 \times 10^{-4} \\
(\varepsilon=0.004)\end{array}$ & $\begin{array}{l}9.92 \times 10^{-4} \\
(\varepsilon=0.02)\end{array}$ & $\begin{array}{l}9.81 \times 10^{-4} \\
(\varepsilon=0.04)\end{array}$ & $\begin{array}{l}9.08 \times 10^{-4} \\
(\varepsilon=0.2)\end{array}$ \\
\hline $7.2 \times 10^{5}$ & $6.17 \times 10^{-2}$ & $6.20 \times 10^{-2}$ & $6.12 \times 10^{-2}$ & $6.18 \times 10^{-2}$ & $6.18 \times 10^{-2}$ & $\begin{array}{l}6.78 \times 10^{-2} \\
(\varepsilon=0.02)\end{array}$ & $\begin{array}{l}6.64 \times 10^{-2} \\
(\varepsilon=0.04)\end{array}$ & $\begin{array}{l}6.29 \times 10^{-2} \\
(\varepsilon=0.2)\end{array}$ & $\begin{array}{l}6.01 \times 10^{-2} \\
(\varepsilon=0.4)\end{array}$ \\
\hline
\end{tabular}

*Two-dimensional prediction (restrained in $\mathrm{X}_{3}$ direction). 
Table 4. Tip deflections of elastic and elastic-plastic beam problem; Fine first-order mesh $(32 \times 192$ elements) elastic-plastic prediction [35]: maximum $=8.22$, residual $=5.28$

\begin{tabular}{|l|c|c|c|}
\hline Element Type & $\begin{array}{l}\text { Elastic } \\
\text { Maximum } \\
\text { Deflection }\end{array}$ & $\begin{array}{l}\text { Elastic-Plastic } \\
\text { Maximum Deflection }\end{array}$ & $\begin{array}{l}\text { Elastic-Plastic } \\
\text { Residual Deflection }\end{array}$ \\
\hline HEX27 & 6.9425 & 7.8994 & 4.3306 \\
\hline HEX27SR & 6.9637 & 8.3775 & 5.4503 \\
\hline WEG21-Mesh-A & 6.9527 & 7.8057 & 4.8879 \\
\hline WEG21R-Mesh-A & 6.9529 & 7.9384 & 4.3281 \\
\hline WEG21SR-Mesh-A & 6.9544 & 7.8604 & 4.6138 \\
\hline WEG21RSR-Mesh-A & 6.9546 & 8.0364 & 4.4005 \\
\hline WEG21LP6-Mesh-A & 6.9637 & 8.0266 & 4.9916 \\
\hline WEG21RLP-Mesh-A & 6.9639 & 8.2590 & 4.4340 \\
\hline WEG21-Mesh-B & 6.9468 & 7.9346 & 5.0779 \\
\hline WEG21R-Mesh-B & 6.9470 & 7.9369 & 5.0866 \\
\hline WEG21SR-Mesh-B & 6.9626 & 8.2378 & 4.9997 \\
\hline WEG21RSR-Mesh-B & 6.9631 & 8.2417 & 5.0061 \\
\hline WEG21LP6-Mesh-B & 6.9591 & 8.1995 & 8.2027 \\
\hline WEG21RLP-Mesh-B & 6.9593 & & \\
\hline
\end{tabular}

Mesh-A, Mesh-B, See Figs. 6. 


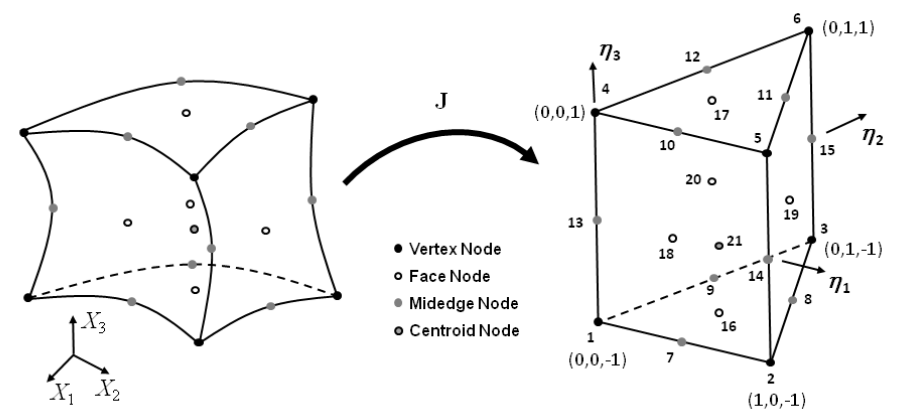

Fig. 1 WEG21 type finite element configuration (left) showing the mapping into its parent element on the right; WEG15 is obtained by omitting the nodes 16-21 on all faces and at the centroid
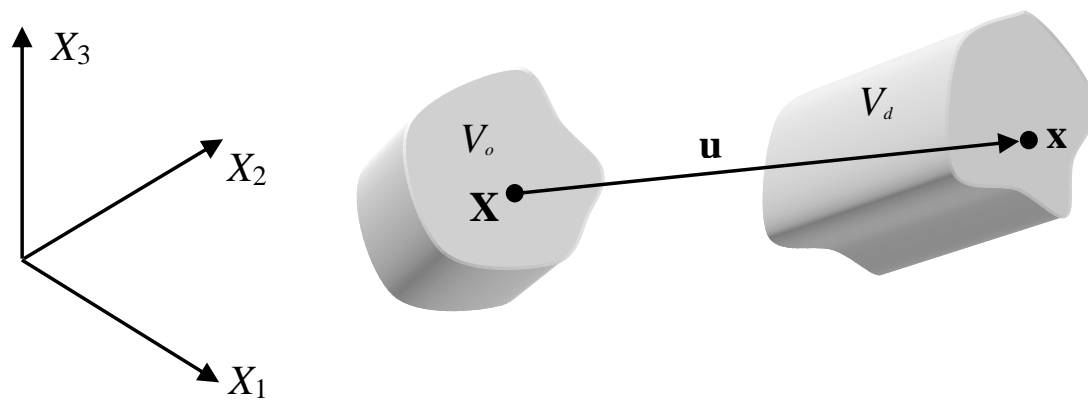

Fig. 2 Arbitrary motion from undeformed to deformed configurations

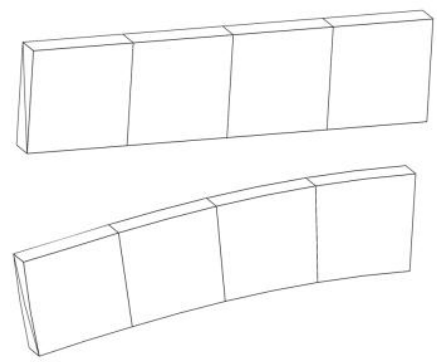

Fig. 3 Half symmetry WEG21 Mesh-1 of uniformly loaded (traction $=7.2 \times 10^{5}$ ) simply supported elastic beam vibration; undeformed (top) and deformed(bottom) at maximum deflection

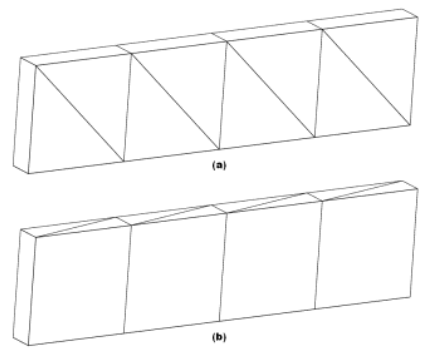

Fig. 4 Alternate arrangement half-symmetry WEG21 models of uniformly loaded simply supported elastic beam vibration (a) Mesh-2 (b) Mesh-3 

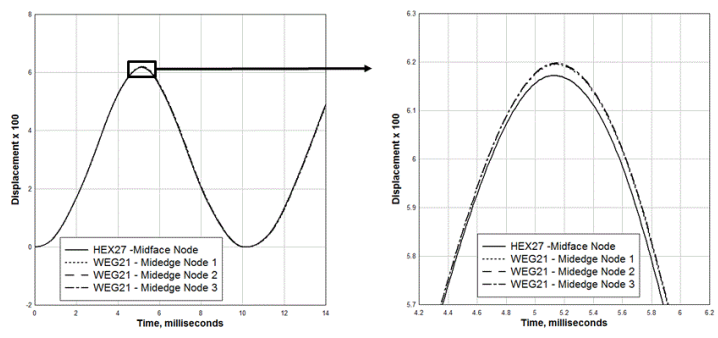

Fig. 5 Midsurface displacement history at midspan of uniformly loaded simply supported elastic beam $\left(\right.$ traction $\left.=7.2 \times 10^{5}\right)$; comparison of different nodes of the WEG21 element (Mesh-1) and HEX27 element
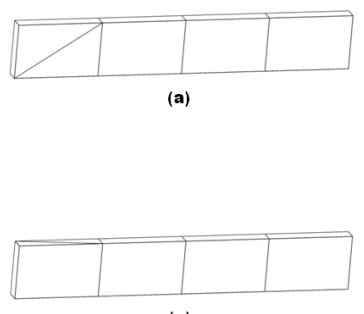

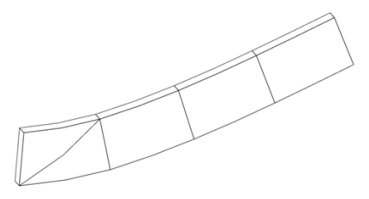

(b)

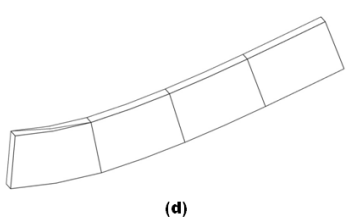

Fig. 6 WEG21RLP models of the tip-shear-loaded cantilevered beam; undeformed and deformed (elastic-plastic) at maximum deflection for (a-b) Mesh-A and (c-d) Mesh-B

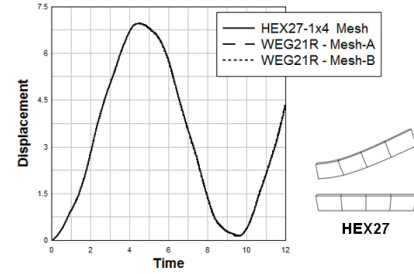

(a)

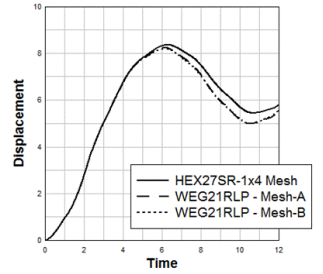

(b)

Fig. 7 End displacement histories of the tip-shear-loaded cantilevered beam comparing different meshes and to HEX27/HEX27SR models (a) WEG21R (elastic) and (b) WEG21RLP (elastic-plastic) 


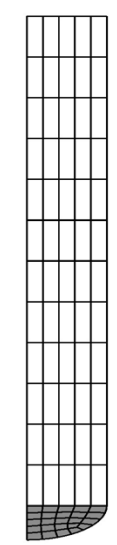

(a)

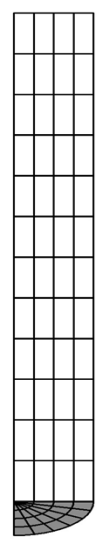

(b)

Fig. 8 Undeformed model plots for bar impact problem using (a) second-order brick (HEX27 type) elements only and (b) a hexahedral-dominant mesh with second-order brick and wedge (HEX27 and WEG21 type) elements

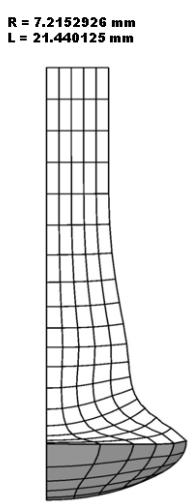

(a)

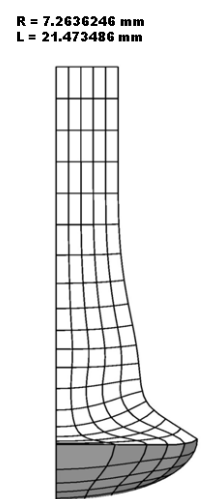

(b)

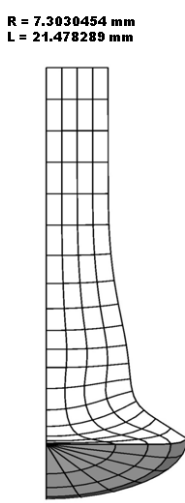

(c)

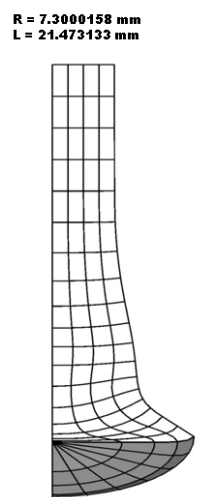

(d)

Fig. 9 Deformed unstructured mesh plots for bar impact problem using (a) HEX27SR elements only, (b) HEX27LP8 elements only, (c) HEX27SR and WEG21SR elements, and (d) HEX27LP8 and WEG21LP6 elements; $\mathrm{R}=$ final radius, $\mathrm{L}=$ final length 


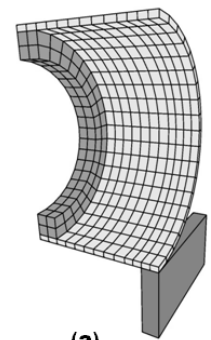

(a)

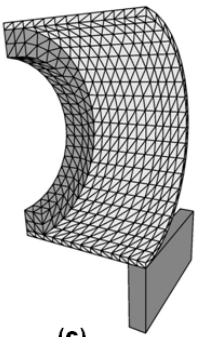

(c)

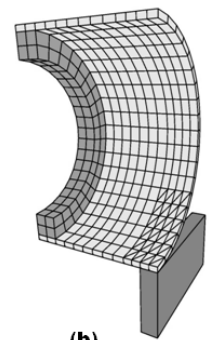

(b)

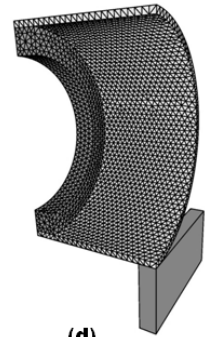

Fig. 10 Quarter-symmetry finite element models for cylinder impact problem; (a) Model-a, HEX27SR elements, (b) Model-b, hexahedral-dominant mesh with HEX27SR and WEG21LP6 elements, (c) Model-c, structured (6 per hex volume) TET15LP4 elements, and (d) Model-d, unstructured TET15LP4 elements

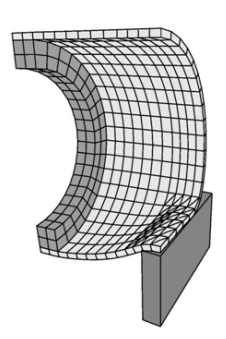

(a)

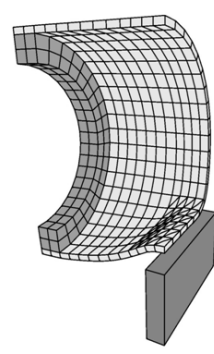

(b)

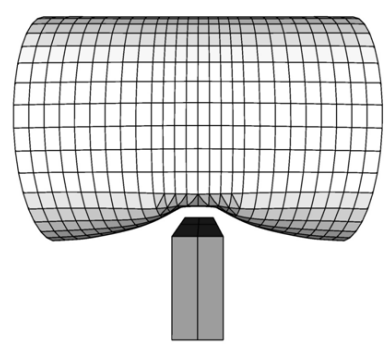

(c)

Fig. 11 Deformation history for cylinder impact problem using Model-b at (a) time of 3 milliseconds, (b) final position-time at 10 milliseconds, and (c) final position-time at 10 milliseconds (full view)

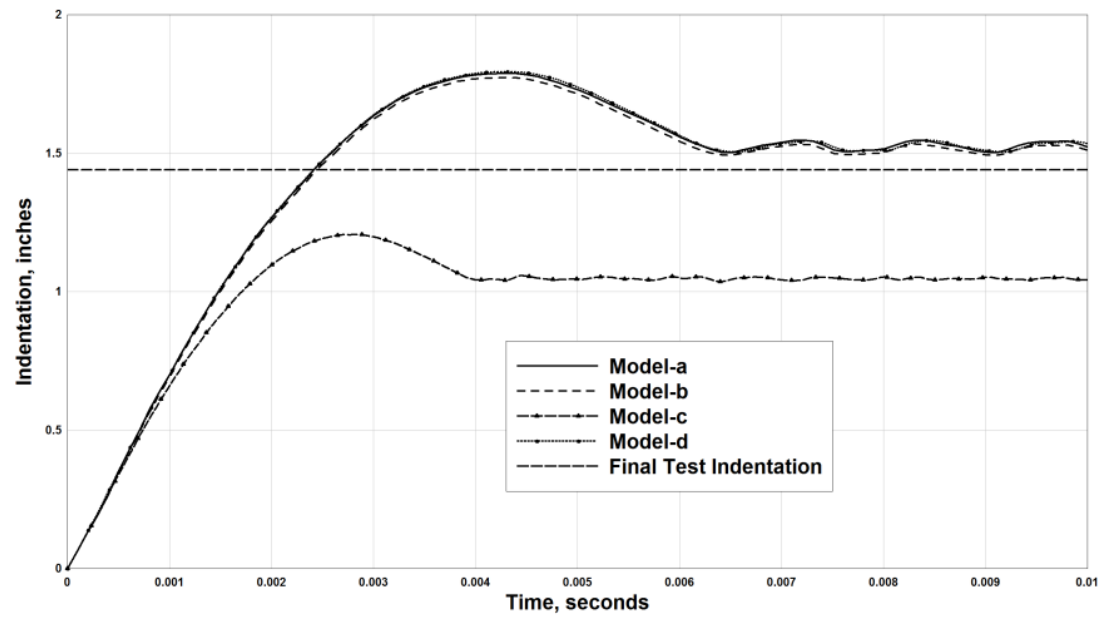

Fig. 12 Maximum model indentation histories for cylinder impact problem 

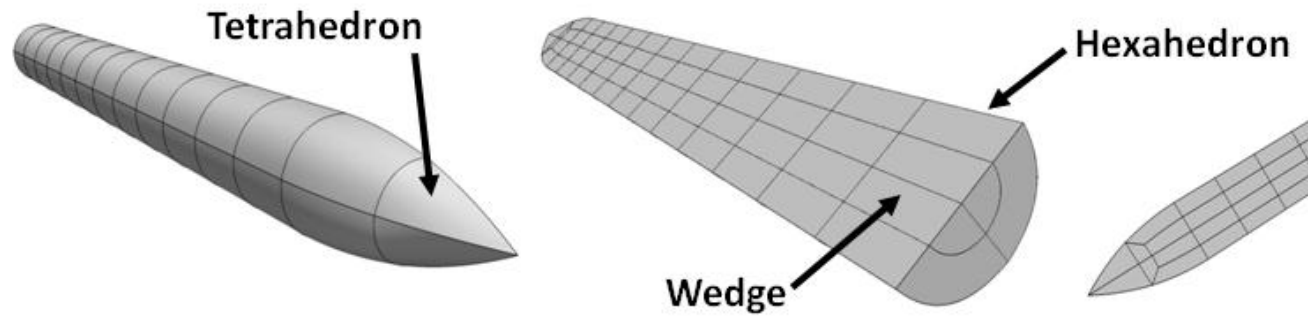

Fig. 13 Half-symmetry Hex-Dominant (HEX27LP8-WEG21LP6-TET15LP4) finite element model for penetrator-concrete impact problem

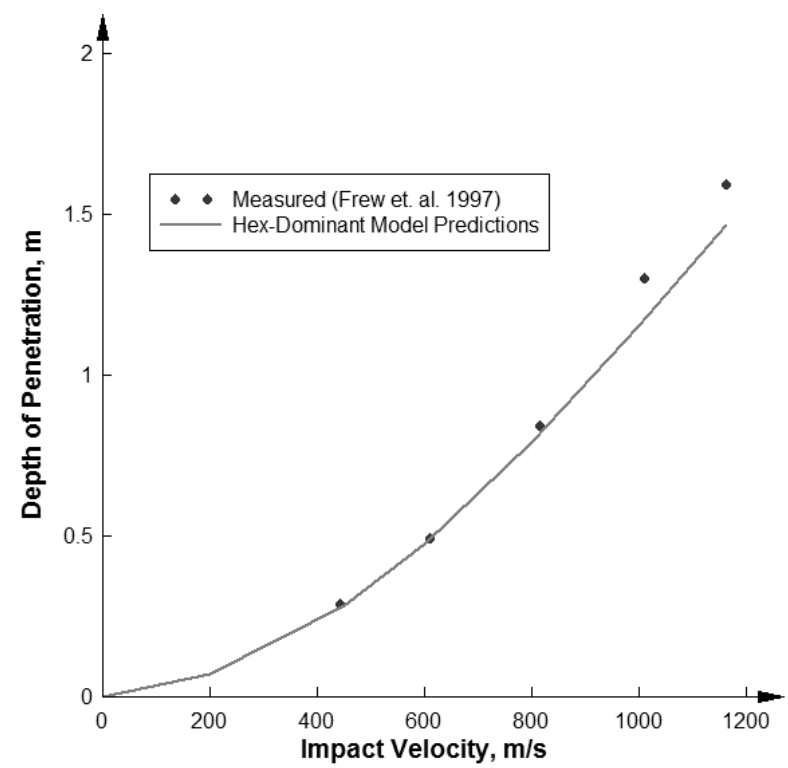

Fig. 14 Penetration depth versus impact velocity for penetrator-concrete impact problem

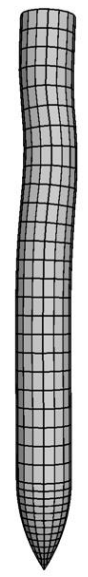

(a)

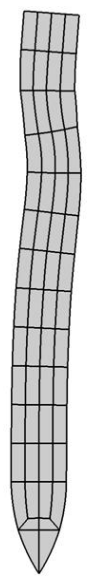

(b)

Fig. 15 Final deformed model plots (impact velocity $=500 \mathrm{~m} / \mathrm{s}$, AOA $=5.0 \mathrm{deg}$.) for (a) Fullsymmetry HEX8FB mesh [40] and (b) Half-symmetry Hex-Dominant mesh 